\title{
AN EXPERIMENTAL ANALYSIS OF MIRROR IMAGE REVERSAL EFFECTS IN PIGEONS
}

\author{
Shigeru Watanabe and Takashi Ogawa
}

\section{Keio University}

Mello (6) reported that pigeons trained monocularly to peck at $45^{\circ}$ tilted line respond maximally to its mirror image, $135^{\circ}$ tilted line, when they were tested for generalization in extinction with the untrained eye. She also found a similar reversal effect after monocular mirror image discrimination $(\mathbf{7}, \mathbf{8})$. Since then this mirror image reversal effect has been examined by several researchers on several species $(5,10)$.

In Mello's experiments, reversal effect occurred both after single stimulus training and mirror image discrimination. However, THOMAS (12) has shown that generalization gradients following binocular single stimulus training using a tilted line have two peaks, i.e., one at the original stimulus and the other at its mirror image. Similar bimodal generalization gradients have been obtained in other binocular training experiments (13) and a monocular training experiment using tilted stripes (14).

One of the purposes of the present experiment is to investigate the difference in generalization gradients after single stimulus training and mirror image discrimination training.

Although the mechanism controlling mirror image reversal effect has not yet been clearly specified, the following two explanations have been suggested so far.

The first is an anatomical or physiological hypothesis based on the results of experiments with optic chiasm sectioned monkeys (10) and the second is a hypothesis formulated in terms of attention in learning $(\mathbf{1}, \mathbf{2})$. In the following we shall be concerned with the adequacy of 
the latter hypothesis.

BEAle and CoOrballis fund that monocularly trained pigeons have a tendency to peck to one particular side of the key, the side of the using eye. They took this fact to mean that pigeons look only at the side of the using eye. Thus if the stimuli are $45^{\circ}$ and $135^{\circ}$ lines, the $45^{\circ}$ line occupies the upper portion on the right side of the key, whereas the $135^{\circ}$ line occupies the lower portion on the same side. This suggests that pigeons may discriminate them on the basis of up-down cue. When the pigons are tested with the untrained eye attending to its side of the key, the cue will be reversed since the attention will have been shifted to the other side.

In support of their hypothesis, Corballis and Beale (4) found that when tested with the trained eye after mirror image discrimination of $45^{\circ}$ and $135^{\circ}$ tilted line, one pigeon trained with the left eye favored the $\otimes$ stimulus while the other two trained with the right eye favored (2) stimulus. But they have not carried out these tests with their untrained eye.

In this experiment we will introduce (c) type stimuli and compare the pigeons' responses to these stimuli after monocular single stimulus training and mirror image discrimination training.

\section{MethoD}

Subjects : Sixteen experimentally naive pigeons maintained at about 80 percent of their free feeding weights.

Apparatus: Modified Skinner box with a single key was used. Grain feeder for reinforcement was attached $15 \mathrm{~cm}$ below the pecking key on which a dark line at various angles was presented by a projector for microscope.

Procedure: All pigeons were binocularly trained to peck a key without any line stimulus by the method of successive approximation. Following pecking training a plastic goggle was mounted with colodion over each of the pigeons' eye to restrict its vision to the anterior field. Then subjects were divided into two groups of eight. One of them, single stimulus training group, was monocularly trained to peck at $135^{\circ}$ tilted line of one $\mathrm{mm}$ width on an illuminated background, while the other, mirror image discrimination group was trained 
also monocularly to peck at $135^{\circ}$ tilted line $\left(\mathrm{S}^{\mathrm{D}}\right)$ but not to peck at $45^{\circ}$ tilted line $\left(\mathrm{S}^{\Delta}\right)$. In either group, half of the subjects were trained with only their right eyes, and the other half were trained with only their left eyes.

In the single stimulus training group, all pigeons received $30 \mathrm{CRF}$ with the $135^{\circ}$ tilted line, one session of FI $10 \mathrm{sec}$ and seven sessions of VI $50 \mathrm{sec}$. Each daily session consisted of five presentations of the $135^{\circ}$ tilted line, one presentation lasting $50 \mathrm{sec}$ with $10 \mathrm{sec}$ blackout. After seven training sessions of VI schedule a generalization test with the trained eye was administered inextinction. The test consisted of eight presentations of eight different angles of tilt ranging from $0^{\circ}$ to $180^{\circ}$ in $22.5^{\circ}$ steps. In each presentation, the stimulus was projected for $25 \mathrm{sec}$ followed by $5 \mathrm{sec}$ blackout period. These stimuli were presented in the order in accordance with $8 \times 8$ Latin square. No reinforcement was available during the testing. On the next day of the generalization test with the trained eye, subjects received one daily training session and were then tested with the untrained eye. After these generalization tests, the same pigeons were subjected to another test with a series of radius stimuli. That is, a $45^{\circ}$ tilted line with its length shortened to the half of the diameter of the pecking key was presented each time on one of the four different portions of the key, i.e., upper left, upper right, lower left, and lower right. As in the generalization test, a stimulus was projected for $25 \mathrm{sec}$ with $5 \mathrm{sec}$ blackout period following. Each of these stimuli was presented four times in accordance with $4 \times 4$ Latin square.

Subjects were tested first with the trained eye and then, after one daily session, with the untrained eye.

In the mirror image discrimination group, one daily session consisted of ten presentations of $\mathrm{S}^{\mathrm{D}}$ and $\mathrm{S}^{\Delta}$ each, alternated in accordance with Gellerman series. During the $\mathrm{S}^{\mathrm{D}}$ period responses were continuously reinforced, while during $\mathrm{S}^{\Delta}$ period no reinforcement was given. This mult CRF-EXT schedule was continued until either 90 percent correct response ratio was reached or 20 sessions of training were over. Then one session of mult FI 12.5 sec-EXT and five sessions of mult VI 25 sec-EXT were given. Following this discrimination training, generalization testing and radius stimuli testing identical to those given to the single stimulus training group were carried out.

\section{RESUlTS}

Fig. 1 presents the individual results of the generalization tests and the radius stimuli tests following the single stimulus training. The 
generalization gradient for each subiect was described as the mean percent of total responses emitted in the presence of each of the test stimuli. Mean of these individual relative gradients are shown in Fig. 2 and Fig. 3.

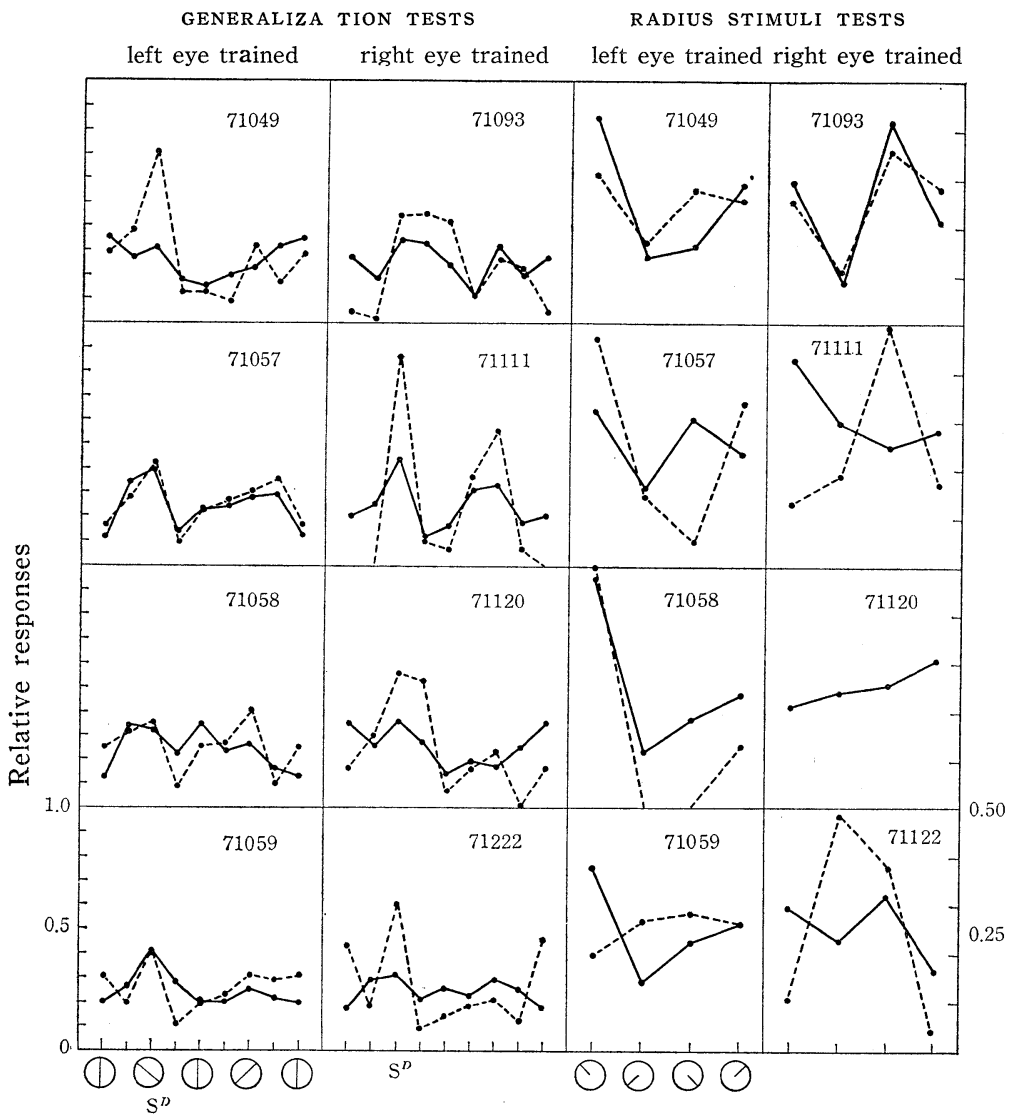

Fig. 1. Responses during generalization tests and radius stimuli tests following the monocular single stimulus training. The solid lines and the broken lines represent the relative gradients with the trained eye and those with the untrained eye respectively. It should be noted that the scale of ordinate for the radius stimuli tests is twice as that for the generalization tests. (Pigeon 71120 emitted no response during the radius stimuli test with the untrained eye.) 
Watanabe - Ogawa : Mirror Image Reversal in Pigeons

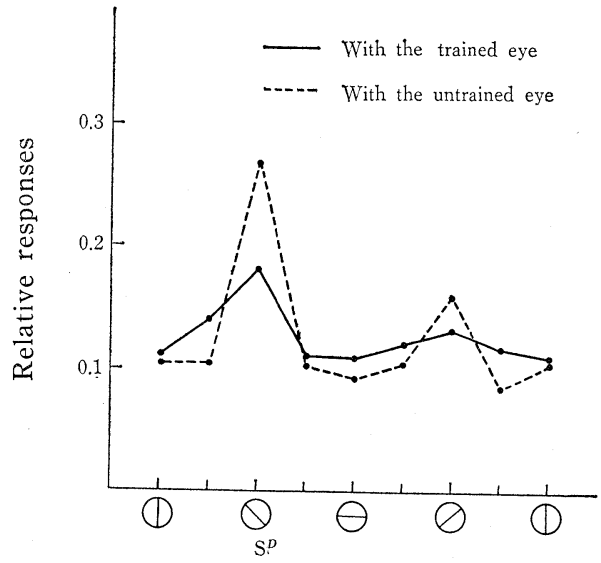

Wig. 2. Mean relative generalization gradients following the monocular single stimulus training.

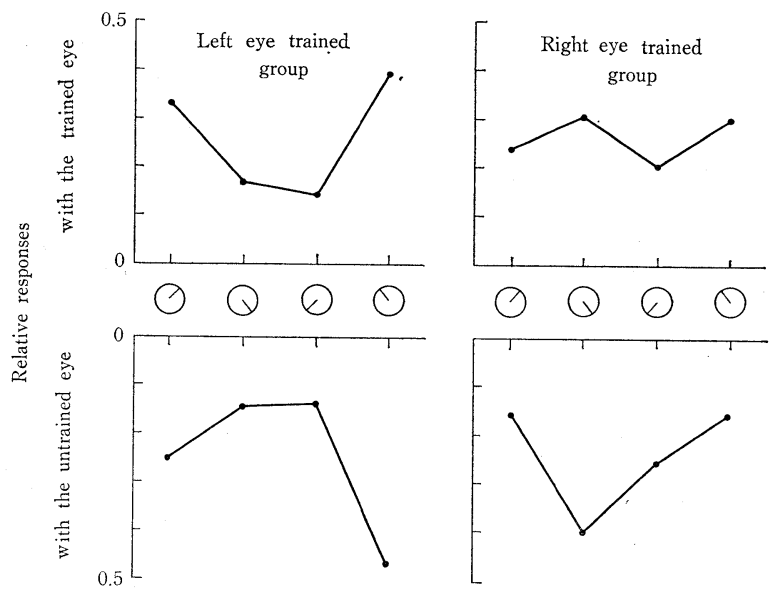

Fig. 3. Mean relative responses during radius stimuli tests after the monocular single stimulus training. The upper part shows the responses with the trained eye; the lower part those with the untrained eye. 
Maximun rates of responding occurs at the original stimulus and its mirror image in the case of the trained eye as well as of the untrained eye. It is seen that all the pigeons responded more to the original stimulus than to its mirror image.

In the generalization tests, performance of the right eye trained subjects were not different from that of the left eye trained ones. However, the results of the radius stimuli tests did make difference depending on which eye was used for training. Subjects trained with their left eye responded maximally at the upper left stimulus and the upper right stimulus, and yet the average gradient by the untrained eye does not show any difference from that by the trained eye.

When tested with the trained eye, two of the right eye untrained subjects $(71093,71122)$ showed the maximum rate of responding at the lower right, 71111 at the upper left, 71120 at the upper left respectively. With the untrained eye, pigeons responded more to the stimuli in the lower portion than to those in the upper portion. Thus, in the case of the right eye trained subjects, the tendency of responding with the trained eye did not agree well with that with the untrained eye. However, it should be noted that contrary relationship, such as the one described later in connection with results of the tests after mirror image discrimination, was not observed between the tests with the trained and the untrained eye.

Fig. 4 shows some typical examples of discrimination learning curves during the mirror image discrimination. Pigeon 71036 in the left eye training group and pigeon 71083 in the right eye training group failed to reach 90 percent criterion. Mean number of the sessions required to attain the criterion was 15 for both subgroups and noticeable difference was not found between them.

Individual results of generalization and radius stimuli tests following discrimination are presented in Fig. 5. The averaged data are shown in Fig. 6 and Fig. 7. With the trained eye open, all subjects maximally responoded at the $\mathrm{S}^{\mathrm{D}}$, whereas with the untrained eye open, five subjects showed the peak of responding at $\mathrm{S}^{\Delta}$, two at $112.5^{\circ}$ and no pigeons 


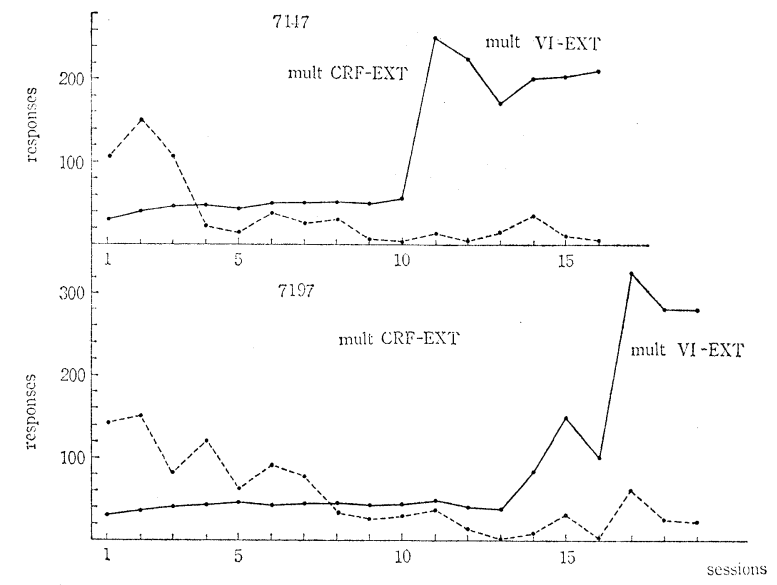

Fig. 4. Examples of discrimination learning curves during the monocular mirror image discrimination. The solid line represents responses emitted for $\mathrm{S}^{\mathrm{D}}$ and the broken line those emitted for $\mathrm{S} \Delta$.

showed the peak at $\mathrm{S}^{\mathrm{D}}$. Correct response ratio during generalization testing, calculated by dividing the number of pecks to $\mathrm{S}^{\mathrm{D}}$ by the sum of the pecks to $\mathrm{S}^{\mathrm{D}}$ and $\mathrm{S}^{4}$, are summarized in Table 1. All pigeons directed more than 85 percent of their pecks to $\mathrm{S}^{\mathrm{D}}$ when they responded with the trained eye. However, with the untrained eye six subjects showed the ratio below 20 percent and the remaining two exhibit the ratio below 40 percent. Thus, the mirror image reversal did occur in all subjects.

In generalization testing there was no difference between the left eye training group and the right eye training group. But in radius stimuli tests the tendency of responding vary with which eye was used for training. As is illustrated in Fig. 5 and Fig. 7, following the left eye training, pigeons maximally responded to the upper left stimulus with the trained eye and to the upper right stimulus with the untrained eye. Thus, the response tendency transferred from the upper left to the upper right depending on the transfer of the eye. On the other hand, pigeons trained with the right eye showed the 
GENERALIZATION TESTS

RADIUS STIMULI TESTS

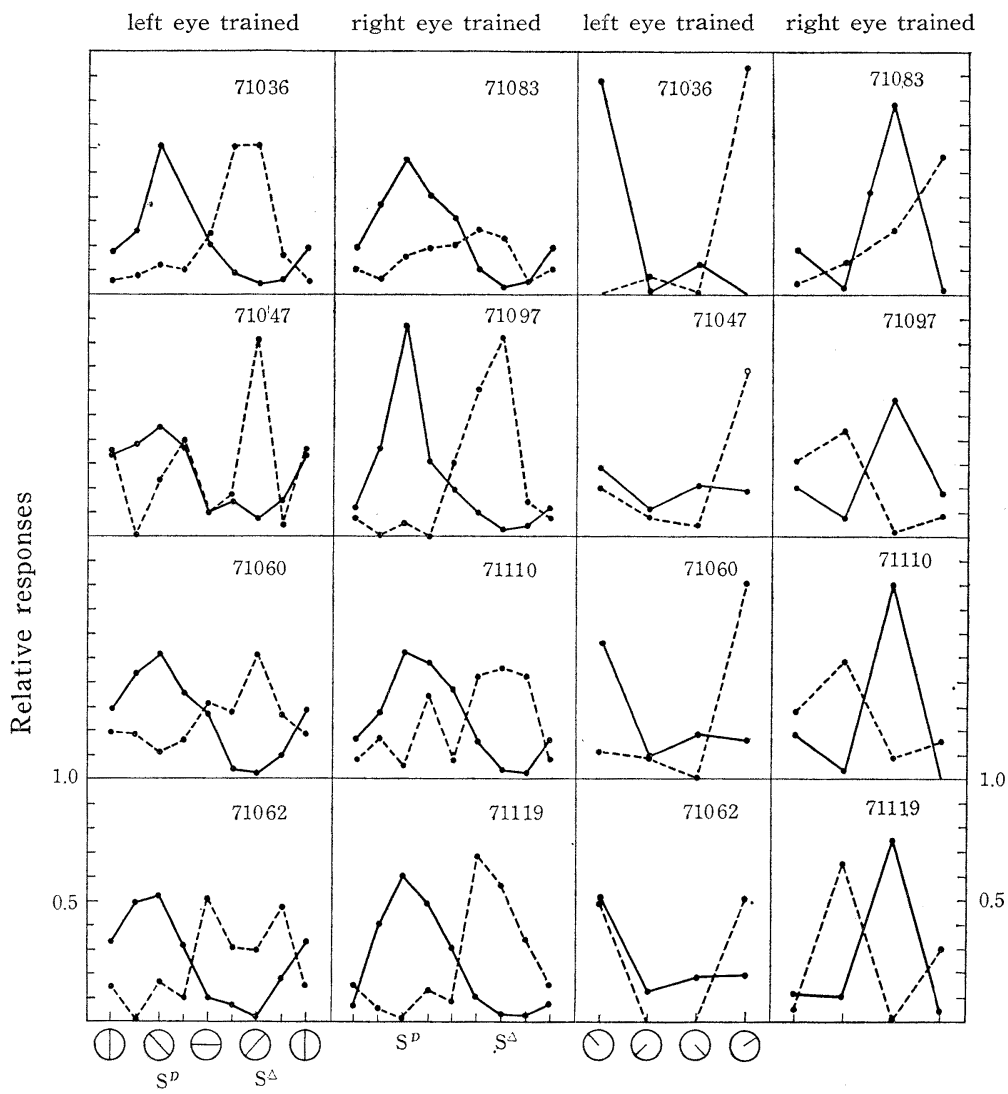

Fig. 5. Responses during generalization tests and radius stimuli tests following the monocular mirror image discrimination. The solid lines represent the relative gradients with the trained eye; the broken lines those with the untrained eye.

peak of responding at the lower right with the trained eye and at the lower left with the untrained eye. The transfer of response tendency was thus from the lower right to the lower left.

\section{Discussion}

Bimodal generalization gradients with peaks at both the trained 


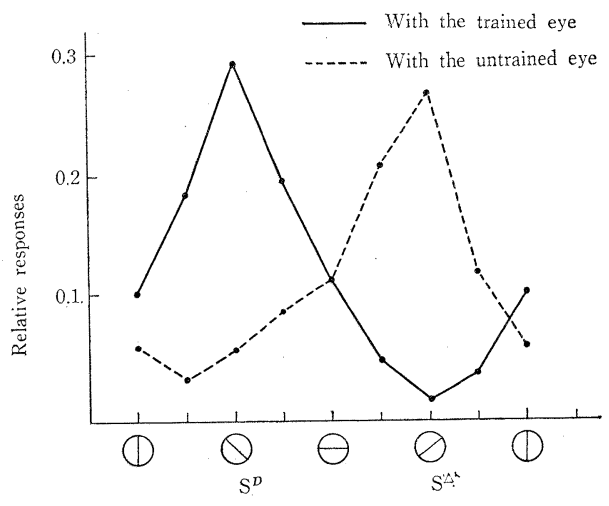

Fig. 6. Mean relative generalization gradient following the monocular mirror image discrimination.

stimulus and its mirror image were obtained after monocular single stimulus training. MELLO obtained the results that the generalization gradients had a single peak at the traintng stimulus for the trained eye and at its mirror image for the untrained eye. The main difference in training procedures in the present experiment and MeLlo's is that she trained pigeons without blackout, whereas we trained pigeons with ten sec blackout. However, Thomas (12) observed bimodal generalization gradient after binocular single stimulus training with and without blackout. Therefore, presence or absence of blackout does not seem to be crucial.

In an experiment on mirror image discrimination Mello (8) found that three out of four pigeons showed the reversal effect. She also reported that for certain mirror image pattern (not line stimulus), there was a lack of interocular transfer of discrimination rather than reversal of responding (7). But using tilted line as stimuli, she invariably observed the reversal effect except for a few unusual subjects.

Contrary to this, Corballis (3) found that only one out of four pigeons showed the reversal effect after monocular training of simultaneous mirror image discrimination. BEALE (2) also obtained 

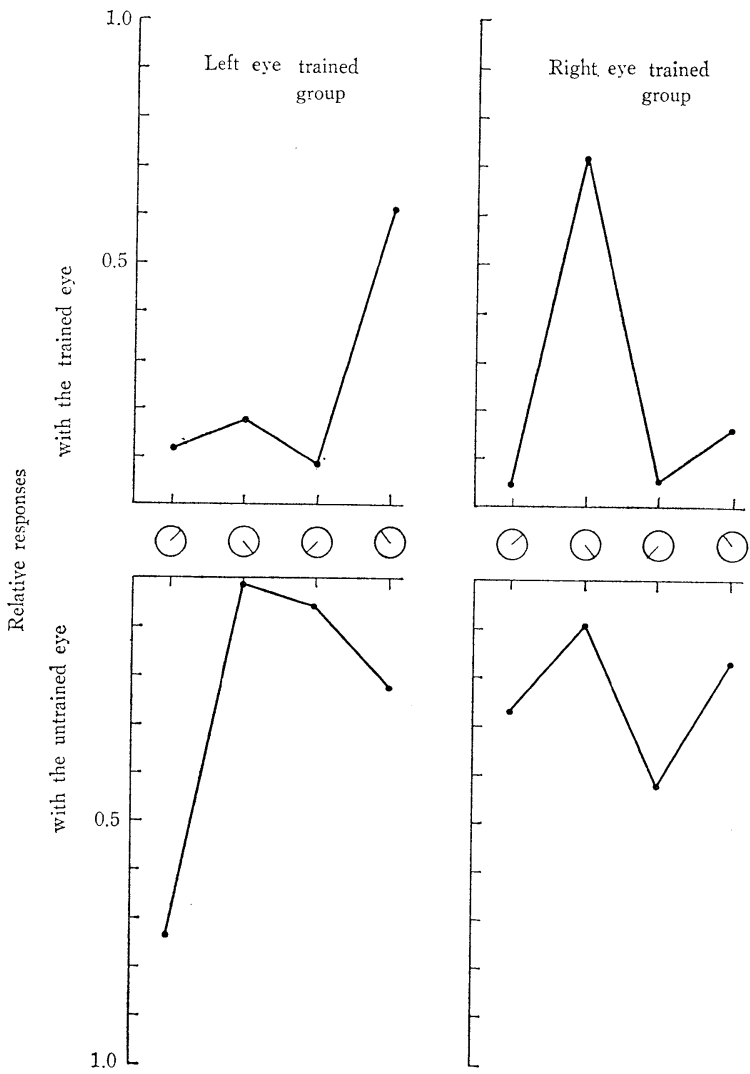

Fig. 7. Mean relative responses during radius stimuli tests after mirror image discrimination. The upper part shows the responses with the trained eye; the lower part those with the untrained eye.

similar results following successive mirror image discrimination. In the present experiment, we have found that the reversal effect are exhibited by all the subjects. It is unclear where the difference in the results obtained here and those of BEALE's comes from, but one may detect a tendency that the longer the period of training was, the more likely the reversal is to occur. 
Table 1. Correct response ratio during generalization tests after the mirror image discrimination.

\begin{tabular}{c|cc}
\hline & TRAINED EYE & UNTRAINED EYE \\
\hline 71036 & .93 & .17 \\
71062 & .96 & .37 \\
71060 & .96 & .18 \\
71047 & .87 & .22 \\
71083 & .95 & .40 \\
71097 & .98 & .06 \\
71110 & .94 & .10 \\
71119 & .97 & .03 \\
\hline
\end{tabular}

Radius stimuli tests following the single stimulus training indicated that pigeons did not differentiate "left" and "right". Pigeons trained with their left eye perceived the $135^{\circ}$ tilted line as in the upper part of the pecking key and when they were subjected to the generalization test, they responded to both the $135^{\circ}$ tilted line and the $45^{\circ}$ tilted line because these stimuli occupied the upper part of the key. Thus, the bimodal generalization gradient can be explained if we assume that pigeons are responding to the "upper" or "lower" part of the stimuli.

The results of the subjects trained with their right eye are ambiguous. However, they responded more to the stimuli in the lower part of the key during the radius stimuli test with their untrained eye. Thus the same idea invoked to explain the generalization gradients by the left eye trained subjects may be adequate also in the case of pigeons trained with the right eye.

However, perceiving the stimuli in a manner like this would bring about totally inappropriate responses if pigeons have been trained to discriminate $135^{\circ}$ tilted line from $45^{\circ}$ tilted one. Subjects trained with their left eye would have to discriminate the upper left from the upper right and those trained with their right eye would have to discriminate the lower left from the lower right. Thus pigeons must bring to bear a different way of discrimination. First assume that they 
discriminate stimuli depending on their using eye. That is, the left eye using subjects respond to the upper left stimulus not because it is on the "left" half of the key but because it is the stimulus presented to them on the side corresponding to their using eye. When they are tested with their untrained eye, what they maximally respond to is the stimulus which contains the component in the upper part of the key corresponing to the using eye, that is, the mirror image of $\mathrm{S}^{\mathrm{D}}$. The results of training with the right eye can be explained in an exactly parallel way.

In summary, our results confirm correctness of an earlier explanation suggested by BEAlE and Corballis $(\mathbf{1}, 2)$ and somewhat clarified the attentional aspects of mirror image reversal effect. However, some cases remain still unexplained and they will be topics of further investigation. For example, this explanation may not hold in the case of interocular transfer of moving direction (9).

\section{SUMMARY}

Pigeons monocularly trained on the mirror image discrimination showed the mirror image reversal effect, but those trained on the single stimulus training showed the bimodal generalization gradients with peaks at $\mathrm{S}^{\mathrm{D}}$ and its mirror image. Tests with radius stimuli indicated that pigeons do not discriminate "left" from "right" during the single stimulus training, whereas they discriminate "left" from "right" on the basis of their using eye in the case of the mirror image discrimination. 


\section{REFERENCES}

1. Beale, I. L., and Corballis, M.C. Laterally displaced pecking in monocularly viewing pigeons: A possible factor in interocular mirror image reversal. Psychon. Sci., 1967, 9, 603-604.

2. Beale, I. L., and Corballis, M.C. Beak shift: an explanation for interocular mirror image reversal in pigeons. Nature, 1968, 220, 82-83.

3. Corballis, M. C., and Beale, I. L. Interocular transfer following simultaneous discrimination of mirror image stimuli. Psychon. Sci., 1967, 9, 605-606.

4. Corballis, M. C., and Beale, I. L. Monocular discrimination of mirror image obliques by pigeons : Evidence for lateralized stimulus control. Anim. Behav., 1970, 18, 563-566.

5. Ingle, D.J. Two visual mechanisms underlying the behavior of fish. Psychol. Forsch., 1967, 31, 44-51.

6. Mello, N. Interhemispheric reversal of mirror image oblique lines after monocular training in pigeons. Sci., 1965, 148, 252-254.

7. Mello, N. Concerning the interhemispheric transfer of mirror image pattern in pigeon. Physiol. \& Behav., 1966, 1, 293-300.

8. Mello, N. Interocular generalization : A study of mirror image reversal following monocular discrimination training in the pigeon. J. exp. Anal. Behav., 1966, 9, 11-16.

9. Mello, N. Interhemispheric transfer of a discrimimation of moving pattern in pigeons. Brain Res., 1968, 7, 390-398.

10. Noble, J. Mirror image and the forebrain commissure of the monkey. Nature, 1966, 211, 1236-1266.

11. Noвle, J. Paradoxial interocular transfer of mirror image discriminations in the optic chiasm sectioned monkey. Brain Res., 1968, 10, 127-151.

12. Thomas, D.R., Klipec, W., and Lyons, J. Investigation of mirror image transfer effect in the pigeons. J. exp Anal. Behav., 1966, 9, 567-570.

13. Vetter, H. G., and Hearst, E. Generalization and discrimination of shape orientation in the pigeon. J. exp. Anal. Behav., 1968, 11, 753-765.

14. Watanabe, S., and Ogawa, T. Interocular transfer in the pigeon : Problem of stimulus modality. The Ann. Anim. Psychol., 1970, 20, 121, (Abstract) "In Japanese". 